\title{
Endoscopic Ultrasound-Guided Gastroenterostomy for Afferent Loop Syndrome
}

\author{
Hideyuki Shiomi', Arata Sakai ${ }^{2}$, Ryota Nakano', Shogo Ota', Takashi Kobayashi', Atsuhiro Masuda ${ }^{2}$ and Hiroko lijima' \\ ${ }^{1}$ Division of Gastroenterology and Hepatobiliary and Pancreatic Diseases, Department of Internal Medicine, Hyogo College of \\ Medicine, Hyogo, ${ }^{2}$ Division of Gastroenterology, Department of Internal Medicine, Kobe University Graduate School of Medicine, Kobe, \\ Japan
}

Afferent loop syndrome (ALS) is a mechanical obstruction of the afferent limbs after gastrectomy with gastrojejunostomy reconstruction. Patients with cancer recurrence require immediate and less invasive treatment because of their poor condition. Percutaneous transhepatic/transluminal drainage (PTD) and endoscopic enteral stenting offer reasonable palliative treatment for malignant ALS but are not fully satisfactory in terms of patient quality of life (QoL) and stent patency. Endoscopic ultrasound-guided gastroenterostomy (EUS-GE) using a lumen-apposing metal stent may address these shortcomings. Clinical data from 11 reports showed that all patients who had undergone EUS-GE had positive technical and clinical outcomes. The adverse event rate was $11.4 \%$, including only mild or moderate abdominal pain, with no severe adverse events. Indirect comparative studies indicated that patients who had undergone EUS-GE had a significantly superior QoL, a higher clinical success rate, and a lower reintervention rate than those who had undergone PTD or endoscopic enteral stenting. Although the evidence is limited, EUS-GE may be considered as a first-line treatment for malignant ALS because it has better clinical outcomes than other less invasive treatments, such as PTD or endoscopic enteral stenting. Further prospective randomized control trials are necessary to establish EUS-GE as a standard treatment for ALS. Clin Endosc 2021;54:810-817

Key Words: Afferent limbs; Afferent loop syndrome; Endoscopic ultrasound; Gastroenterostomy; Lumen-apposing metal stent

\section{INTRODUCTION}

Afferent loop syndrome (ALS) is a late complication of Billroth II procedures and other gastrojejunostomies and is defined as a mechanical obstruction of the afferent limbs. ${ }^{1}$ It can be classified as benign or malignant depending on the cause of the obstruction. The incidence of benign ALS appears to have decreased with advances in surgery, whereas that of ma-

\footnotetext{
Received: September 14, 2021 Revised: October 15, 2021

Accepted: October 17, 2021

Correspondence: Hideyuki Shiomi

Division of Gastroenterology and Hepatobiliary and Pancreatic Diseases, Department of Internal Medicine, Hyogo College of Medicine, 1-1 Mukogawa-cho, Nishinomiya, Hyogo 663-8501, Japan

Tel: +81-798-45-6472, Fax: +81-798-45-6474, E-mail: hideshio0403@gmail.com ORCID: https://orcid.org/0000-0002-7461-8538

It is the invited review article.
}

(cc) This is an Open Access article distributed under the terms of the Creative Commons Attribution Non-Commercial License (http://creativecommons.org/ licenses/by-nc/3.0) which permits unrestricted non-commercial use, distribution, and reproduction in any medium, provided the original work is properly cited. lignant ALS has increased despite advances in chemotherapy. ${ }^{2}$ Cholangitis, pancreatitis, and perforation due to increased intraluminal pressure may occur, leading to rapidly progressing and severe disease requiring immediate treatment. Surgical management has been widely applied; however, it seems to be undesirable for patients with advanced oncological settings because it may cause further morbidity and mortality by delaying and interrupting oncological treatment. ${ }^{3}$ Thus, patients with a poor condition and limited prognoses tend to prefer less invasive treatments. The efficacy of endoscopic enteral self-expandable metal stent (SEMS) placement as an alternative treatment to surgery has been reported in recent studies. ${ }^{4-8}$ However, potential limitations include a relatively high reintervention rate and challenging SEMS placement in cases of long strictures or angulated afferent limbs. ${ }^{9}$

Recently, various therapeutic endoscopic ultrasound (EUS) techniques have been developed and have significantly contributed to the treatment of pancreatobiliary diseases. EUS-guided gastroenterostomy (EUS-GE), a therapeutic EUS modality, has attracted attention as a minimally invasive 
treatment for gastric outlet obstruction. ${ }^{10}$ It is an innovative bypass technique that involves deploying a metal stent without passing through the intestinal stricture with the scope using a lumen-apposing metal stent (LAMS), which has further improved the efficacy and safety. However, data on EUS-GE for ALS remain limited. In this review article, we provide an overview of EUS-GE for ALS, especially malignant ALS.

\section{AFFERENT LOOP SYNDROME}

The afferent loop is a segment of the small intestine that is located proximal to a gastrojejunostomy or jejunojejunostomy site after gastrectomy with Billroth II or Roux-en-Y reconstruction and a Whipple procedure; it consists of the distal duodenum or proximal jejunum, also known as the afferent limb. ALS is defined as a mechanical obstruction of the afferent limb. ${ }^{3}$ The etiologies of the mechanical obstruction are as follows: (i) postoperative adhesions, internal herniation, volvulus, and intussusception, (ii) stenosis due to ulceration at the gastrojejunostomy site, (iii) radiation enteritis of the afferent loop, (iv) locoregional cancer recurrence, and (v) enteroliths, bezoars, and foreign bodies impacted in the afferent loop.

The symptoms of ALS are associated with increased intraluminal pressure due to biliary, pancreatic, and enteric secretion accumulation in a closed loop following partial and complete afferent limb obstruction. ${ }^{11}$ The main symptoms are abdominal distension, upper abdominal pain, nausea, and vomiting due to dilatation of the afferent limbs. In addition, the outflow of pancreatic juice and bile is obstructed, resulting in pancreatitis and ascending cholangitis. Patients who have undergone a Whipple procedure have been reported to be more susceptible to pancreaticobiliary symptoms because of the loss of papillary function. ${ }^{12}$ In severe cases, bowel distention and ischemia may lead to intestinal necrosis, perforation, and peritonitis, subsequently leading to the development of sepsis due to the translocation of overgrowing bacteria into the systemic circulation. Therefore, early diagnosis and treatment are necessary before the disease becomes severe.

The incidence of ALS after distal gastrectomy with Billroth II or Roux-en-Y reconstruction has been reported to range from $0.2 \%$ to $1.0 \% .{ }^{13}$ Pannala et al. ${ }^{1}$ evaluated the incidence of ALS in patients with pancreatic cancer who had undergone a Whipple procedure. In their report, $13 \%$ of patients developed ALS at a median follow-up duration of 1.2 years after surgery. Among those with ALS, the incidence of obstruction due to recurrent pancreatic cancer was 33\%.

\section{Current management of ALS (Table 1)}

The management of ALS depends on whether the cause of the obstruction is benign or malignant. If benign lesions cause ALS, surgery is selected as the first choice for curative treatment. ${ }^{14}$ If malignant lesions cause ALS, drainage is selected as a palliative approach, since curative treatment through surgery is not feasible, and the patient's condition is often poor. Percutaneous transhepatic/transluminal drainage (PTD) is the mainstay treatment for malignant ALS. PTD options include percutaneous transhepatic biliary drainage $(\mathrm{PTBD})^{15}$ and direct percutaneous transluminal drainage of the dilated afferent limbs. ${ }^{16}$ These treatments are minimally invasive and reasonable for the treatment of malignant ALS. However, the disadvantages include a reduction in quality of life owing to the presence of an external fistula and the risk of serious complications, such as bacteremia due to ascending cholangitis, ${ }^{17}$ hemobilia, intraperitoneal hemorrhage, and biliary peritonitis.

Recent advances in endoscopic interventions have provided

Table 1. Management of Afferent Loop Syndrome

1. Percutaneous drainage

(a) Direct percutaneous drainage

(b) Percutaneous transhepatic biliary drainage

2. Endoscopic drainage

(a) Endoscopic balloon dilatation

(b) Endoscopic enteral plastic stent placement

(c) Endoscopic enteral metal stent placement

3. Endoscopic ultrasound-guided drainage

(a) Endoscopic ultrasound-guided gastroenterostomy

(b) Endoscopic ultrasound-guided biliary drainage 
novel alternative therapeutic options. Endoscopic approaches include enteral double-pigtail or metal stent placement or balloon dilatation. ${ }^{18}$ In the retrospective study by Pannala et al., 15 of 24 (62\%) patients with malignant ALS after a Whipple procedure for pancreatic cancer underwent endoscopic intervention. Among these patients, 11 (73\%) had successful clinical outcomes. However, recurrence occurred in $75 \%$ of patients who had received plastic stent placement, requiring PTBD or percutaneous rendezvous-assisted biliary stenting. Therefore, in malignant ALS, SEMS placement is preferred over balloon dilatation and plastic stent placement to avoid recurrence. Sakai et al. ${ }^{19}$ reported a case series of seven patients who underwent endoscopic enteral SEMS placement for malignant ALS after pancreaticoduodenectomy (PD). In all cases, subtotal stomach-preserving PD was performed for primary malignant diseases, including five cases of pancreatic cancer, one case of duodenal cancer, and one case of bile duct cancer. The endoscopic procedure involved the use of a therapeutic gastrointestinal endoscope (GIF 1T260; Olympus Optical Co., Tokyo, Japan) with a 3.7-mm working channel. All patients had successful clinical outcomes, with no adverse events. Moreover, all patients died of their primary disease, and the median overall survival period was 155 (range, 96-374) days. No stent dysfunction was observed in these patients prior to their death. These results indicate that endoscopic treatment with enteral SEMS placement is effective and safe for the treatment of malignant ALS.

Recently, a balloon-assisted endoscope (BAE) with a 3.2-mm working channel, which is capable of enteral SEMS placement using the through-the-scope method, has become available. This type of BAE is helpful for enteral SEMS placement if the conventional scope cannot reach the stenotic site. ${ }^{4-7}$ Furthermore, an additional SEMS placement can be performed if SEMS occlusion occurs. Therefore, enteral SEMS placement using a BAE with a larger working channel is an effective, less invasive, and promising treatment for malignant ALS and may become the first choice of treatment. However, endoscopic enteral SEMS placement across the stricture is sometimes challenging because of the long enteric segment, long stricture segment, and acute angulation. An alternative approach for the treatment of malignant ALS is then needed to overcome these limitations.

\section{Indications for EUS-GE in patients with ALS}

EUS-GE involves creating a bypass pathway by anastomosing the stomach and enteric walls using a stent under EUS. It may be advantageous in treating patients with malignant ALS because the stent does not pass through the enteral stricture. Previously, EUS-GE with pigtail stent placement has been performed; however, this technique may involve complications, such as intraoperative and postoperative leakages. ${ }^{20}$ However, the recent availability of LAMSs, which consist of a fully covered metal stent with bilateral anchor flanges, has facilitated further development of EUS-GE to become a safer and more effective procedure for ALS, with a potentially high success rate and decreased risk of leakage and stent migration. ${ }^{9}$

EUS-GE can be performed if the distance from the EUS transducer to the afferent limbs is within $1 \mathrm{~cm} .{ }^{21}$ However, it is not indicated for patients undergoing endoscopic procedures when coagulopathy, intervening vessels, and unstable conditions are present. For ascites, EUS-GE can be performed in cases involving small amounts of fluid but should be avoided in massive ascites because of poor fistula creation, which can lead to peritonitis and infection (Table 2). ${ }^{22}$

\section{EUS-GE technique for ALS}

For successful EUS-GE, it is important to accurately assess the location of the stomach and afferent limbs using axial and coronal computed tomographic images as a roadmap. In addition, the collateral vessels and ascites should be checked to avoid complications.

Under EUS guidance, the dilated afferent limbs are identified from the stomach. If the distance from the stomach to the afferent limbs is estimated to be $>1 \mathrm{~cm}$, the procedure should

Table 2. Indications for Endoscopic Ultrasound-Guided Gastroenterostomy in Patients with Afferent Loop Syndrome

Indications

The distance from the endoscopic ultrasound transducer to the afferent limbs is $<1 \mathrm{~cm}$.

Contraindications

Severe coagulopathy

Intervening vessel

Unstable conditions unfit for an endoscopic procedure

Massive ascites 
be discontinued or switched to an alternative treatment, such as endoscopic enteral stenting or PTD. The dilated afferent limbs are punctured using a $19-\mathrm{G}$ needle. The contrast medium is injected through the needle following aspiration of the fluid content. A 0.025 - or 0.035 -inch stiff guidewire is then placed into the dilated afferent limbs, and a fistula is dilated using a balloon catheter or mechanical or electrocautery dilators. Finally, an LAMS is deployed from the dilated afferent limbs to the stomach. This technique has been widely used as a classical multistep procedure. ${ }^{9}$ An electrocautery-enhanced LAMS (EC-LAMS) has recently been developed, which involves an attachment to a cautery wire at the tip of the delivery system. It enables puncture, dilatation, and deployment of the stent in a single step, facilitating the procedure, shortening the procedure time, and reducing the risk of leakage (Fig. 1). ${ }^{23,24}$
LAMSs have the advantage of preventing stent migration and fluid leakage. However, they are unavailable in many countries and cost more than conventional fully covered self-expandable metal stents (FCSEMSs). Shiomi et al. ${ }^{25}$ developed a modified stent system to prevent stent migration and fluid leakage, similar to an LAMS. This method involves the deployment of an FCSEMS with antimigration functions; it consists of a large-loop double-pigtail plastic stent within an FCSEMS (Fig. 2).

\section{Clinical outcomes of EUS-GE in patients with ALS}

Table 3 summarizes previous reports concerning EUSGE for ALS. ${ }^{9,20,23-31}$ The reports were identified from the PubMed database for English-language studies using the following keywords: "afferent loop syndrome" and "endo-
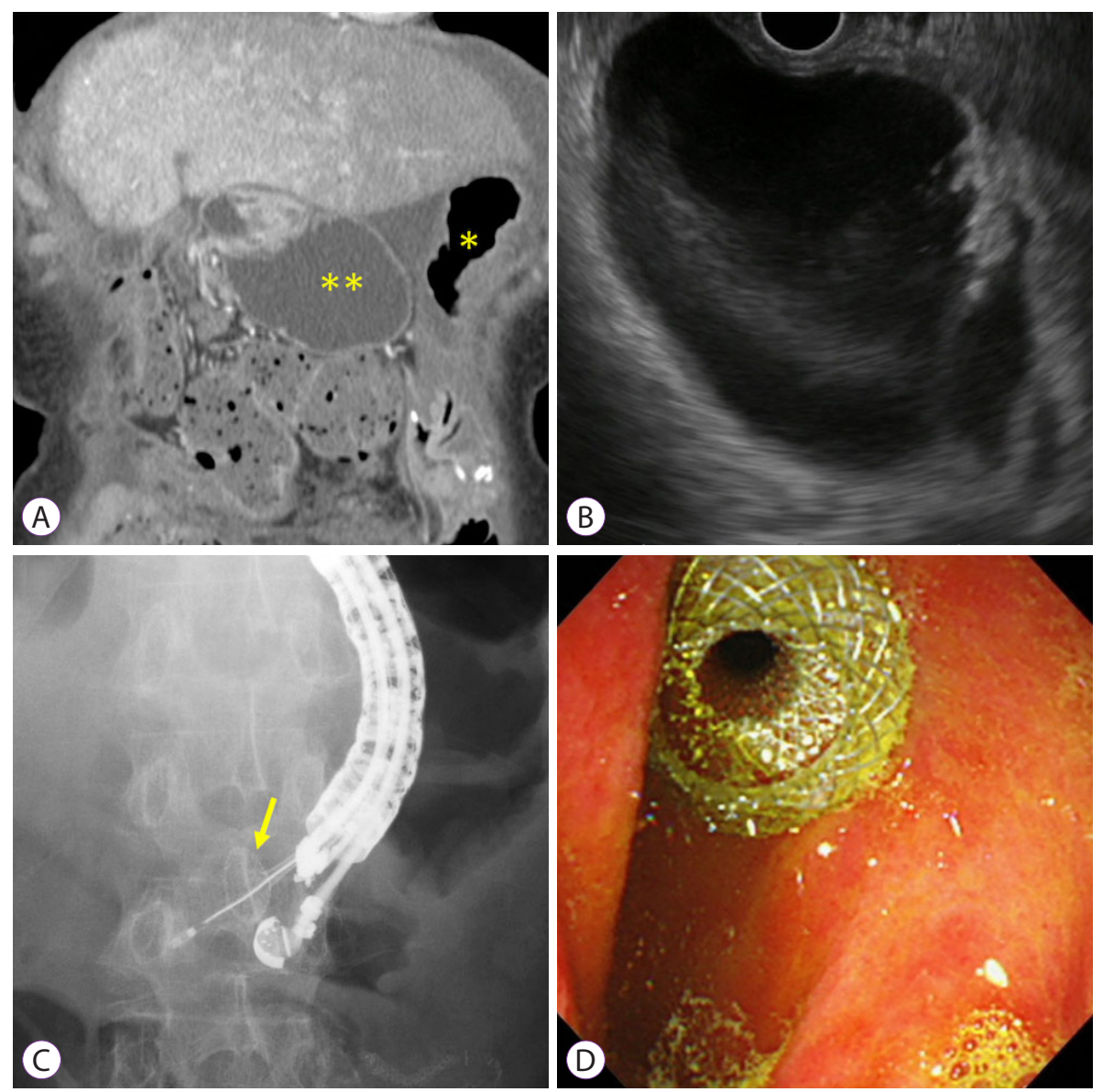

Fig. 1. Endoscopic ultrasound-guided gastroenterostomy using an EC-LAMS. (A) Contrast-enhanced computed tomography reveals that the dilated afferent limbs are associated with a recurrence of pancreatic cancer $\left({ }^{*}\right.$, stomach; ${ }^{* *}$, dilated afferent limbs). (B) EUS shows dilatation of the afferent limbs from the stomach. (C) The dilated afferent limbs are punctured using an EC-LAMS, and the distal flange is then deployed under EUS guidance (yellow arrow: distal flange). (D) The proximal flange is deployed under endoscopic imaging. EC-LAMS, electrocautery-enhanced lumen-apposing metal stent; EUS, endoscopic ultrasound. 

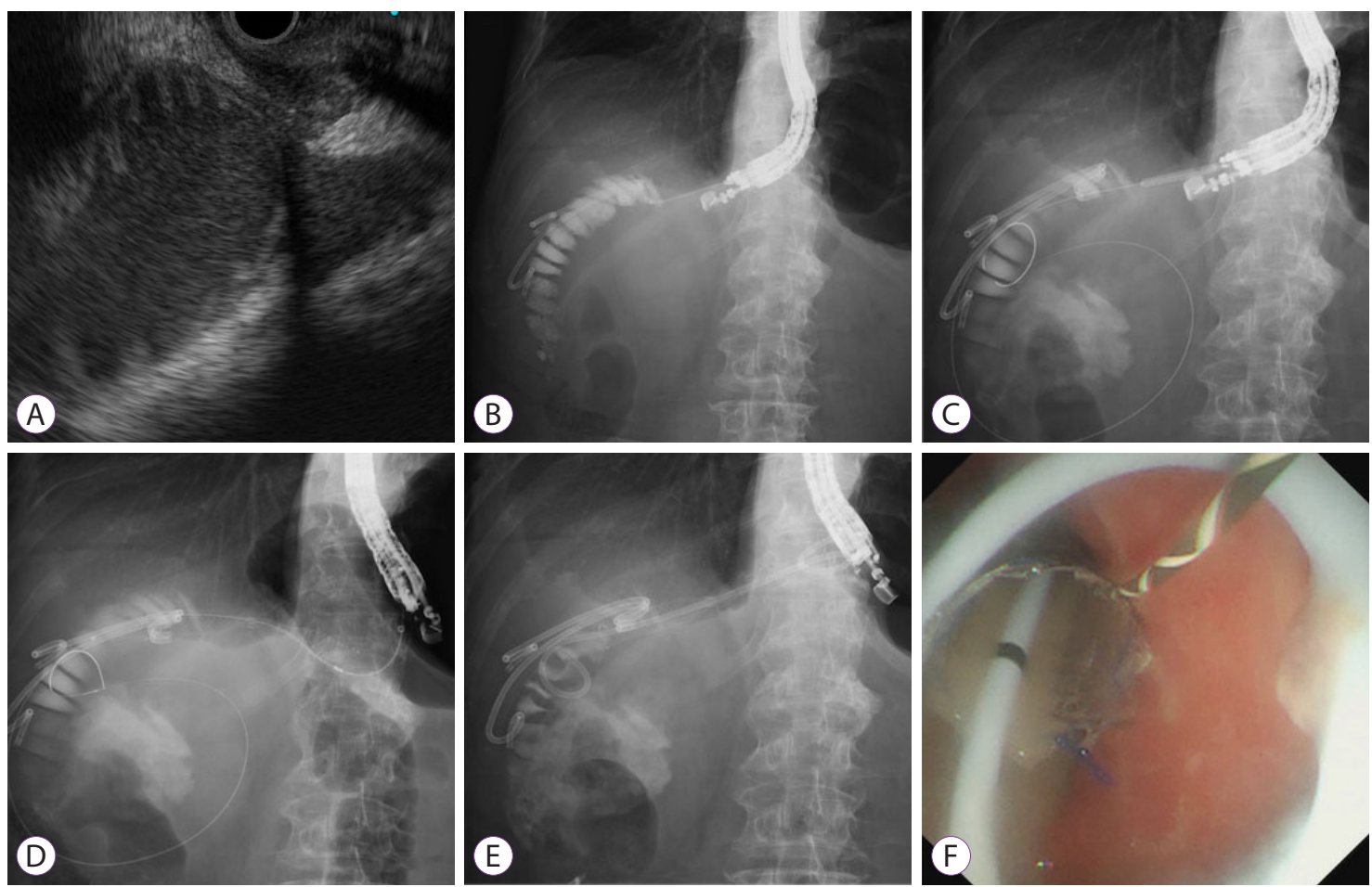

Fig. 2. Endoscopic ultrasound-guided gastroenterostomy using a fully covered self-expandable metal stent combined with a large loop double-pigtail stent. (A) On the endoscopic ultrasound image, the dilated afferent limbs can be visualized from the stomach. (B) The dilated afferent limbs are punctured using a 19-G needle, and a contrast medium is injected. (C) A guidewire is inserted to the afferent limbs, and the fistula is dilated using a balloon catheter. (D) A fully covered self-expandable metal stent is deployed to appose the afferent limbs and stomach. (E, F) A large loop double-pigtail plastic stent is placed through the fully covered self-expandable metal stent.

Table 3. Outcomes of Endoscopic Ultrasound-Guided Gastroenterostomy in Patients with Afferent Loop Syndrome

\begin{tabular}{|c|c|c|c|c|c|c|}
\hline Study & $\mathbf{N}$ & Drainage method & Stent & $\begin{array}{l}\text { Technical } \\
\text { success }\end{array}$ & $\begin{array}{l}\text { Clinical } \\
\text { success }\end{array}$ & Adverse events \\
\hline Matsumoto et al. $(2013)^{20}$ & 1 & EUS-GJ & Pigtail PS & 1 & 1 & 0 \\
\hline Ikeuch et al. $(2015)^{24}$ & 1 & EUS-GJ & EC-LAMS & 1 & 1 & 0 \\
\hline Shah et al. $(2015)^{27}$ & 1 & EUS-GJ & LAMS & 1 & 1 & 0 \\
\hline Taunk et al. $(2015)^{26}$ & 3 & EUS-GJ & LAMS & 3 & 3 & 0 \\
\hline Yamamoto et al. $(2017)^{23}$ & 1 & EUS-GJ & EC-LAMS & 1 & 1 & 0 \\
\hline Brewer et al. $(2018)^{9}$ & 18 & $\begin{array}{l}\text { EUS-GJ, 13; } \\
\text { EUS-JJ, } 5\end{array}$ & $\begin{array}{l}\text { EC-LAMS, 12; } \\
\text { LAMS, } 6\end{array}$ & 18 & 18 & Abdominal pain, 3 \\
\hline Benallal et al. $(2018)^{29}$ & 1 & EUS-GJ & LAMS & 1 & 1 & Abdominal pain, 1 \\
\hline Sakamoto et al. $(2019)^{30}$ & 1 & EUS-GJ & Tubular SEMS & 1 & 1 & 0 \\
\hline Lajin et al. $(2019)^{31}$ & 1 & EUS-GJ & EC-LAMS & 1 & 1 & 0 \\
\hline Shiomi et al. $(2019)^{25}$ & 1 & EUS-GJ & Tubular SEMS + pigtail PS & 1 & 1 & 0 \\
\hline Bie et al. $(2021)^{28}$ & 6 & EUS-GJ & EC-LAMS & 6 & 6 & 0 \\
\hline Overall & 35 & & & $\begin{array}{c}100 \% \\
(35 / 35)\end{array}$ & $\begin{array}{c}100 \% \\
(35 / 35)\end{array}$ & $11.4 \%(4 / 35)$ \\
\hline
\end{tabular}

EC-LAMS, electrocautery-enhanced LAMS; EUS-GJ, endoscopic ultrasound-guided gastrojejunostomy; EUS-JJ, endoscopic ultrasound-guided jejunojejunostomy; LAMS, lumen-apposing metal stent; N, number; PS, plastic stent; SEMS, self-expandable metal stent. 
scopic ultrasound-guided gastrojejunostomy"/"endoscopic ultrasound-guided gastroenterostomy"/"endoscopic ultrasound-guided entero-enterostomy." We reviewed the clinical data of 35 patients, derived from nine case studies and two retrospective studies. The overall technical and clinical success rate was $100 \%$. The adverse event rate was $11.4 \%(n=4)$, and all four patients experienced abdominal pain (mild, $n=3$; moderate, $n=1$ ). No severe adverse events, such as bleeding, peritonitis, or perforation, were observed. Thirty-two of the thirty-five patients were treated with LAMS placement. Of these, 21 underwent a one-step deployment of an EC-LAMS. These results indicate that EUS-GE may be an effective and safe technique for treating ALS. However, publication bias was possible because the number of cases was small, and the procedures were performed only by experts. Therefore, further trials involving a larger number of patients are required.

\section{Comparison with other less invasive palliative therapies}

Few previous studies have compared EUS-GE with other less invasive therapies, including PTD and endoscopic enteral stenting. Brewer et al. ${ }^{9}$ reported the findings of their retrospective comparative study of EUS-GE and endoscopic enteral stenting in patients with malignant ALS. They indirectly compared the multicenter data of patients treated with EUS-GE $(n=18)$ with single-center data of patients treated with endoscopic enteral stenting $(n=17)$. The rate of clinically successful outcomes was reported to be significantly higher in patients who had undergone EUS-GE than in those who had undergone endoscopic enteral stenting in terms of complete symptom resolution ( $88.9 \%$ vs. $5.9 \% ; P<0.001)$. There was no significant difference in the technical success rate (100\% vs. $88.2 \% ; P=0.23)$, adverse event rate $(16.7 \%$ vs. $5.9 \% ; P=0.67)$, or procedure time (29.7 vs. 41.6 minutes; $P=0.13$ ) between the two groups. However, the rate of the need for reintervention was significantly lower in the EUS-GE group than in the endoscopic enteral stenting group $(16.7 \%$ vs. $76.5 \%$; $P<0.001)$. These results indicated that EUS-GE had a higher clinical success rate and a lower reintervention rate than endoscopic enteral stenting. Since EUS-GE has the potential advantage of drainage without crossing the stricture of the afferent limbs, it may yield a more favorable outcome than endoscopic enteral stenting.

Bie et al. ${ }^{28}$ conducted a retrospective comparative study between PTD and EUS-guided drainage, including EUS-GE and EUS-guided hepaticogastrostomy (EUS-HGS) for malignant ALS. Their study included 17 patients, five of whom underwent PTD, six underwent EUS-GE, and six underwent EUSHGS. Technical success was achieved in all approaches. The clinical success rate was not significantly different between the
PTD, EUS-GE, and EUS-HGS groups ( $80 \%$ vs. $100 \%$ vs. $100 \%$, respectively, $P=0.455)$. However, the rate of bilirubin level decrease was significantly higher in patients who underwent EUS-guided drainage than in those who underwent PTD. Adverse events only occurred in patients who underwent PTD $(60 \%, P=0.015)$. Moreover, the number of reinterventions was lower in patients who underwent EUS-guided drainage than in those who underwent PTD (0 [interquartile range, $0.0-1.0$ ]) vs. 1 [0.5-2.5], respectively; $P=0.045)$. The clinical outcomes of EUS-GE and EUS-HGS were comparable in their study. Based on these results, the authors concluded that EUS-guided drainage may be preferable to PTD for malignant ALS. In addition, EUS-HGS may be a feasible alternative salvage procedure when the EUS-GE technique is challenging.

\section{Future perspectives}

With advances in imaging and the development of effective adjuvant chemotherapies, surgery at an early stage has improved the prognosis of patients with cancer after surgery. However, the incidence of ALS is increasing; therefore, a less invasive and long-term patency procedure to relieve ALS is required. Recently, with the advent of LAMSs, favorable data on EUS-GE for ALS have been reported, although the number of reports is limited. ${ }^{9,23,24,26-29,31}$ Furthermore, the availability of EC-LAMSs has enabled single-step deployment, and EUS-GE has become a simpler and safer procedure. EUS-GE has the advantage of ensuring long-term stent patency because it does not involve crossing the stricture of the afferent limbs, allowing for continuous effective chemotherapy, which is expected to prolong survival.

EUS-GE is not usually performed in patients with ascites. However, recent reports have shown that patients with mild or localized ascites could be considered for EUS-GE without risking leakage or peritonitis if the ascites does not interfere with the drainage route. Therefore, the presence of ascites should not be considered a strict contraindication for EUS-GE, except for massive ascites. ${ }^{9,2}$ Despite limited evidence, we believe that EUS-GE should be the first choice of treatment for ALS if the distance between the dilated afferent limbs and the stomach is close and if there is no intervening ascites based on the results of previous reports. ${ }^{9,2}$ However, if massive ascites is present, endoscopic enteral SEMS placement should be considered to prevent severe complications associated with EUS-GE. Furthermore, more invasive treatments, such as PTD or surgery, are recommended when these endoscopic treatments fail or are not fully effective.

EUS-GE in patients with surgically altered anatomy has been applied not only in the treatment of ALS but also in the performance of endoscopic retrograde cholangiopancreatography (ERCP) in patients who have undergone Roux-en-Y 
gastric bypass (RYGB). In recent years, RYGB has been commonly used as a treatment for obesity. When pancreatobiliary diseases, such as common bile duct stones, occur in patients who have undergone RYGB, balloon enteroscopy-assisted ERCP (BE-ERCP) is selected as a less invasive treatment, which is technically challenging. ${ }^{32}$ A novel treatment method has been developed to overcome this challenge; in this method, a fistula is created between the remnant and excluded stomach under EUS guidance, and conventional ERCP is then performed via the fistula, which is known as EUS-guided gastrogastrostomy-assisted ERCP (EUS-GG-ERCP). ${ }^{33,34} \mathrm{~A}$ retrospective study that compared between EUS-GG-ERCP and BE-ERCP in 60 patients with an RYGB anatomy (EUS-GGERCP group, $n=30$; BE-ERCP group, $n=30$ ) was conducted by Bukhari et al. ${ }^{34}$ In that study, the EUS-GG-ERCP group had a higher technical success rate $(100 \%$ vs. $60.0 \%, P<0.001)$ and a shorter procedure time than the BE-ERCP group (49.8 vs. 90.7 minutes, $P<0.001)$. Meanwhile, the adverse event rate was comparable between the groups. In the future, a combined treatment with EUS-GE and ERCP is likely to be applied to pancreatobiliary diseases complicated by ALS.

\section{CONCLUSIONS}

Although evidence remains limited, EUS-GE appears to be a promising minimally invasive treatment option for ALS, especially malignant ALS. Furthermore, the availability of ECLAMSs has made EUS-GE a simpler and safer procedure. EUS-GE may be considered as a first-line therapy in patients with ALS because it yields better clinical outcomes than do other less invasive treatments, such as endoscopic enteral SEMS placement or PTD. To date, some case reports and a limited number of retrospective studies have been published; however, no prospective studies have been conducted. Therefore, further verified prospective randomized control trials are necessary to establish EUS-GE as a standard treatment for ALS.

Conflicts of Interest

The authors have no potential conflicts of interest.

Funding

This work was supported by the JSPS KAKENHI (Grants-in-Aid for Scientific Research; grant number 21K07913 to H.S.).

\section{Acknowledgments}

We wish to thank Professor Takao Itoi and Takayoshi Tsuchiya from Tokyo Medical University for providing the images.
Author Contributions

Conceptualization: Hideyuki Shiomi

Formal analysis: HS, Arata Sakai

Supervision: Hiroko Iijima

Visualization: HS, Ryota Nakano, Shogo Ota

Writing-original draft: HS

Writing-review\&editing: Takashi Kobayashi, Atsuhiro Masuda

ORCID
Hideyuki Shiomi:

Arata Sakai:

Ryota Nakano:

Shogo Ota:

Takashi Kobayashi:

Atsuhiro Masuda:

Hiroko Iijima: https://orcid.org/0000-0002-7461-8538 https://orcid.org/0000-0003-1072-5243 https://orcid.org/0000-0002-1844-2865 https://orcid.org/0000-0001-5171-1847 https://orcid.org/0000-0002-9202-7643 https://orcid.org/0000-0002-2138-1207 https://orcid.org/0000-0002-5841-8827

\section{REFERENCES}

1. Pannala R, Brandabur JJ, Gan SI, et al. Afferent limb syndrome and delayed GI problems after pancreaticoduodenectomy for pancreatic cancer: single-center, 14-year experience. Gastrointest Endosc 2011;74:295302.

2. Song KB, Yoo D, Hwang DW, et al. Comparative analysis of afferent loop obstruction between laparoscopic and open approach in pancreaticoduodenectomy. J Hepatobiliary Pancreat Sci 2019;26:459-466.

3. Blouhos K, Boulas KA, Tsalis K, Hatzigeorgiadis A. Management of afferent loop obstruction: reoperation or endoscopic and percutaneous interventions? World J Gastrointest Surg 2015;7:190-195.

4. Yane K, Katanuma A, Hayashi T, et al. Enteral self-expandable metal stent placement for malignant afferent limb syndrome using single-balloon enteroscope: report of five cases. Endosc Int Open 2018;6:E1330-E1335.

5. Sasaki T, Yamada I, Matsuyama M, Sasahira N. Enteral stent placement for malignant afferent loop obstruction by the through-the-scope technique using a short-type single-balloon enteroscope. Endosc Int Open 2018;6:E806-E811.

6. Shimatani M, Takaoka M, Tokuhara M, et al. Through-the-scope self-expanding metal stent placement using newly developed short double-balloon endoscope for the effective management of malignant afferent-loop obstruction. Endoscopy 2016;48(Suppl 1 UCTN):E6-E67.

7. Minaga K, Kitano M, Takenaka M. Through-the-scope enteral metal stent placement using a short-type single-balloon enteroscope for malignant surgically reconstructed jejunal stenosis (with video). Dig Endosc 2016;28:758

8. Kanno Y, Ohira T, Harada Y, et al. Metal stent placement in the afferent loop obstructed by peritoneal metastases-experience of five cases. Clin Endosc 2018;51:299-303.

9. Brewer Gutierrez OI, Irani SS, Ngamruengphong S, et al. Endoscopic ultrasound-guided entero-enterostomy for the treatment of afferent loop syndrome: a multicenter experience. Endoscopy 2018;50:891-895.

10. Tonozuka R, Tsuchiya T, Mukai S, Nagakawa Y, Itoi T. Endoscopic ultrasonography-guided gastroenterostomy techniques for treatment of malignant gastric outlet obstruction. Clin Endosc 2020;53:510-518.

11. Sakai A, Shiomi H, Masuda A, Kobayashi T, Yamada Y, Kodama Y. Clinical management for malignant afferent loop obstruction. World J Gastrointest Oncol 2021;13:684-692.

12. Termsinsuk P, Chantarojanasiri T, Pausawasdi N. Diagnosis and treatment of the afferent loop syndrome. Clin J Gastroenterol 2020;13:660668.

13. Grisé K, McFadden D. Anastomotic technique influences outcomes af- 
ter partial gastrectomy for adenocarcinoma. Am Surg 2001;67:948-950.

14. Bolton JS, Conway WC. Postgastrectomy syndromes. Surg Clin North Am 2011;91:1105-1122.

15. Kim KH, Lee HB, Kim SH, Kim MC, Jung GJ. Role of percutaneous transhepatic biliary drainage in patients with complications after gastrectomy. Int Surg 2015 May 29 [Epub]. https://doi.org/10.9738/INTSURG-D-15-00117.1.

16. Sato Y, Inaba Y, Murata S, et al. Percutaneous drainage for afferent limb syndrome and pancreatic fistula via the blind end of the jejunal limb after pancreatoduodenectomy or bile duct resection. J Vasc Interv Radiol 2015;26:566-572.

17. Moriura S, Ikeda S, Kimura A, Iwatsuka Y, Ikezawa T, Naiki K. Jaundice due to afferent loop obstruction following hepatectomy for a hilar cholangiocarcinoma. Abdom Imaging 1996;21:226-227.

18. Kim HJ, Park JY, Bang S, Park SW, Lee YC, Song SY. Self-expandable metal stents for recurrent malignant obstruction after gastric surgery. Hepatogastroenterology 2009;56:914-917.

19. Sakai A, Shiomi H, Iemoto T, et al. Endoscopic self-expandable metal stent placement for malignant afferent loop obstruction after pancreaticoduodenectomy: a case series and review. Clin Endosc 2020;53:491496.

20. Matsumoto K, Kato H, Tomoda T, et al. A case of acute afferent loop syndrome treated by transgastric drainage with EUS. Gastrointest Endosc 2013;77:132-133.

21. Wannhoff A, Ruh N, Meier B, Riecken B, Caca K. Endoscopic gastrointestinal anastomoses with lumen-apposing metal stents: predictors of technical success. Surg Endosc 2021;35:1997-2004.

22. Itoi T, Baron $\mathrm{TH}$, Khashab MA, et al. Technical review of endoscopic ultrasonography-guided gastroenterostomy in 2017. Dig Endosc 2017;29:495-502.

23. Yamamoto K, Tsuchiya T, Tanaka R, et al. Afferent loop syndrome treated by endoscopic ultrasound-guided gastrojejunostomy, using a lumen-apposing metal stent with an electrocautery-enhanced delivery system. Endoscopy 2017;49:E270-E272.

24. Ikeuchi N, Itoi T, Tsuchiya T, Nagakawa Y, Tsuchida A. One-step EUS-guided gastrojejunostomy with use of lumen-apposing metal stent for afferent loop syndrome treatment. Gastrointest Endosc 2015;82:166.

25. Shiomi H, Kobayashi T, Sakai A, et al. Endoscopic ultrasound-guided gastrojejunostomy using fully covered metal stent combined with largeloop double-pigtail stent for malignant afferent loop syndrome. Endoscopy 2019;51:E303-E304.

26. Taunk P, Cosgrove N, Loren DE, Kowalski T, Siddiqui AA. Endoscopic ultrasound-guided gastroenterostomy using a lumen-apposing self-expanding metal stent for decompression of afferent loop obstruction. Endoscopy 2015;47(Suppl 1 UCTN):E395-E396.

27. Shah A, Khanna L, Sethi A. Treatment of afferent limb syndrome: novel approach with endoscopic ultrasound-guided creation of a gastrojejunostomy fistula and placement of lumen-apposing stent. Endoscopy 2015;47(Suppl 1 UCTN):E309-E310.

28. De Bie C, Bronswijk M, Vanella G, et al. EUS-guided hepaticogastrostomy for patients with afferent loop syndrome: a comparison with EUS-guided gastroenterostomy or percutaneous drainage. Surg Endosc 2021 Apr 28 [Epub]. https://doi.org/10.1007/s00464-021-08520-z.

29. Benallal DC, Hoibian S, Caillol F, et al. EUS-guided gastroenterostomy for afferent loop syndrome treatment stent. Endosc Ultrasound 2018;7:418-419.

30. Sakamoto Y, Hijioka S, Maruki Y, et al. Endoscopic ultrasound-guided gastroenterostomy using a metal stent for the treatment of afferent loop syndrome. Endoscopy 2019;51:E153-E155.

31. Lajin M, Catalano MF, Khan NM, Lajin I. Endoscopic ultrasound-guided gastrojejunostomy using a 2-cm lumen-apposing metal stent to treat benign afferent loop syndrome. Endoscopy 2019;51:695-696.

32. Skinner M, Popa D, Neumann H, Wilcox CM, Mönkemüller K. ERCP with the overtube-assisted enteroscopy technique: a systematic review. Endoscopy 2014;46:560-572.

33. Kedia P, Sharaiha RZ, Kumta NA, Kahaleh M. Internal EUS-directed transgastric ERCP (EDGE): game over. Gastroenterology 2014;147:566568.

34. Bukhari M, Kowalski T, Nieto J, et al. An international, multicenter, comparative trial of EUS-guided gastrogastrostomy-assisted ERCP versus enteroscopy-assisted ERCP in patients with Roux-en-Y gastric bypass anatomy. Gastrointest Endosc 2018;88:486-494. 